\title{
Intraoperative nerve identification and chronic postherniorrhaphy pain
}

\author{
H. Kehlet • J. M. Bischoff • E. K. Aasvang • \\ M. U. Werner
}

Received: 16 August 2012/Accepted: 2 September 2012/Published online: 18 September 2012

(C) Springer-Verlag 2012

First of all, we thank Drs. Chen and Amid [1] for their very thoughtful comments and update on the role of intraoperative nerve identification during groin hernia repair and the risk of development of chronic pain. We agree with all their comments, except that our conclusions in the abstract does not "conclude that nerve identification does not decrease rates of pain in routine practice" as quoted, but rather that "although intraoperative inguinal nerve identification should be aimed at, other factors may contribute to the risk of nerve damage and persistent pain after open groin hernia repair" [2]. This conclusion supports the comments by Chen and Amid [1], despite that we in the present study were not able to demonstrate any influence of nerve identification on signs of nerve damage or on development of persistent pain. The discussion on identification of the "genitofemoral nerve" (which we entirely agree should be named "genital branch") is to some extent semantic as we agree that routine direct identification should not be done, but only if necessary for the herniotomy per se. This may explain our low identification rate of the genital branch $(21.3 \%)$ which, interestingly, is within the same range as reported from a recent hernia specialist publication on persistent pain problems, with an identification rate of $26.5 \%$ [3]. Also, we agree that the use of heavy-weight mesh may have contributed to the pain problems, but when the study was conducted there was little conclusive evidence for differences between mesh type and persistent pain.

In summary, the valuable comment by Drs. Chen and Amid provides a sound balance on this important (but also difficult) topic of persistent postherniorrhaphy pain compared to our rather negative findings [1]. We entirely support their conclusion that surgical technique is a crucial component to reduce this disturbing problem.

\section{References}

1. Chen DC, Amid PK (2012) Questioning the favorable influence of nerve identification during open inguinal herniorrhaphy is misleading and may increase the risk of surgeons' liability. Hernia. doi: 10.1007/s10029-012-0967-5

2. Bischoff JM, Aasvang EK, Kehlet H, Werner MU (2012) Does nerve identification during open inguinal herniorrhaphy reduce the risk of nerve damage and persistent pain? Hernia. doi: 10.1007/ s10029-012-0946-x

3. Campanelli G, Pascual MH, Hoeferlin A, Rosenberg J, Champault G, Kingsnorth A, Miserez M (2012) Randomized, controlled, blinded trial of Tisseel/Tissucol for mesh fixation in patients undergoing Lichtenstein technique for primary inguinal hernia repair: results of the TIMELI trial. Ann Surg 255:650-657

H. Kehlet · J. M. Bischoff $(\bowtie) \cdot$ E. K. Aasvang

Section of Surgical Pathophysiology 4074,

Rigshospitalet, Copenhagen University,

Blegdamsvej 9, 2100 Copenhagen, Denmark

e-mail: jomutahi@hotmail.com

J. M. Bischoff · M. U. Werner

Multidisciplinary Pain Centre 7612, Rigshospitalet,

Copenhagen University, Copenhagen, Denmark 